\title{
Observing atomic resolution dynamics of soft materials with controlling dose rate
}

Fu-Rong Chen ${ }^{1}$, Dirk Van Dyck ${ }^{2}$, Christian Kisielowski ${ }^{3}$ and Stig Helveg ${ }^{4}$

${ }^{1}$ MSE department, City University of Hong Kong, United States, ${ }^{2}$ EMAT, Department of Physics, University of Antwerp, Belgium, United States, ${ }^{3}$ Lawrene Berkeley National Laboratory, The Molecular Foundry and Joint center for Artifical Photosynthesis, University of California, Berkeley, United States, ${ }^{4}$ Center for Visualizing Catalytic Processes (VISION), Department of Physics, Technical University of Denmark, DK2800 Kgs. Lyngby, Denmark., Kongens Lyngby, Hovedstaden, Denmark

Advancements of aberration-corrected electron optics and data acquisition schemes have made TEM capable of delivering images with sub-Ångström resolution and single-atom sensitivity. However, three bottlenecks, namely, radiation damage, static imaging and 2D projection geometry are still challenging TEM imaging of soft materials at the atomic level. In the present paper, we present advancements of electron microscopy methodology towards probing 3D atomic resolution dynamics at high temporal resolution and emphasize in particularly the role of controlling the electron delivery.

For the radiation damage, it has been shown that the electron beam may not only alter the shape and surface structure at atomic level $[1,2]$, but also induce radiolytic artifacts in chemical reactions at nanoscale, e.g., photocatalysis, metal-insulator transformation (MIT), super-dissolution of oxides $[3,4,5]$. Two examples of electron beam effects at nanometer resolution are shown in the Fig. 1. Fig. 1a, 1b, and 1c shows a dose-rate dependence of hydrogen bubble generation accompanying with water dissociation of $\mathrm{Pt}$ loaded $\mathrm{TiO}_{2}$ nanoparticles in a liquid cell during in situ TEM observations. It clearly shows that the in-situ observations of photocatalysis can really mix up with radiolysis from the electron beam. An insulator phase of $\mathrm{VO}_{2}$ is known to transform to metal phase at $68^{\circ} \mathrm{C}$. Fig. $1 \mathrm{~d}$ shows that a nano probe at high electron dose-rate can stimulate phase transformation from insulator phase to metal phase in a $\mathrm{VO}_{2}$ nanowire even at room temperature. But the electron delivery effect can even be addressed at atomic resolution, as shown in Fig. 2. Fig. 2a, b and c show an example of modulus and phase of an exit wave of a $\mathrm{MoS}_{2}$ nanoparticle which were quantitatively analyzed using new analytical model, to determine $S$ vacancy dynamics.

The dose-rate of incident electrons becomes a very critical and is inevitable a degree of freedom that must be controlled to surpress radiolysis artifacts and to reveal pristine structure at atomic resolution. In our approach, to overcome three imaging bottlenecks simultaneously, the electron dose was evenly distributed onto many different focal planes with pulse electron source operated in low dose-rate mode. With an aberration-corrected TEM, the focal series of images at atomic-resolution can be recorded with $100 \mathrm{~ms} /$ frame at low dose-rate electron illumination and used to reconstruct in-line electron holograms with a high signal/noise ratio even with a one second time resolution. From the holograms, atomic structure in 3D, including the atomic position and atom content can be retrieved $[6,7]$. To further minimize the electron beam effect and boost a higher time resolution on atomic resolution dynamics of fragile materials, for example, MOF , carbon nanotube and molecules at atomic level, the use of a pulse electron source is beneficial. Some new experimental results from a new aberration corrected TEM equipped with electron pulse source and fast camera will be reported. Similar to the cryoEM for protein 3D reconstruction, a novel expectation maximum algorithm, for example, simulation annealing is also development that allow to recover 3D atomic resolution dynamics at near nanosecond regime will be discussed. 

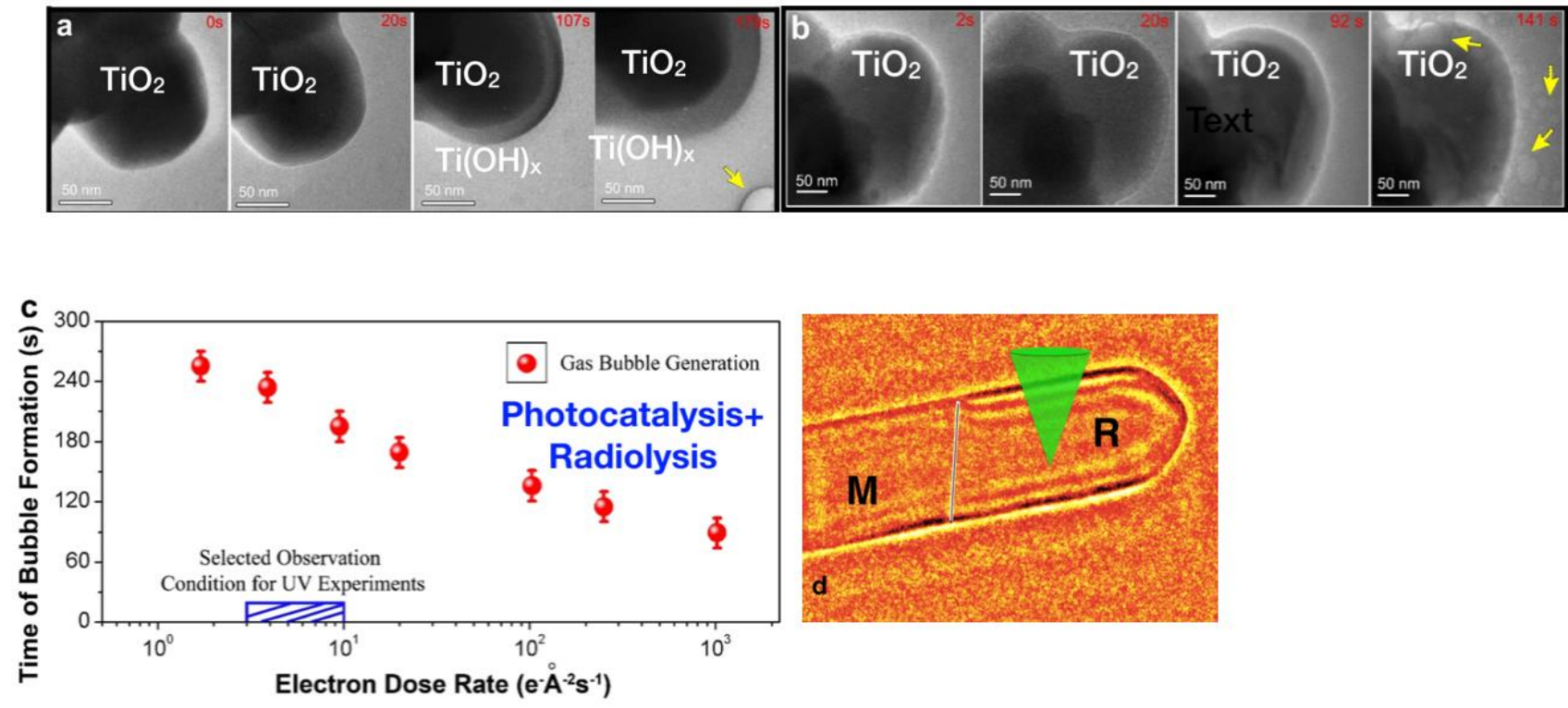

Figure 1. Pt loaded TiO2 nanoparticles in a liquid cell. These particle was illuminate with UV for in-situ TEM observation, dose rate (a) 20e/A2 $2 \mathrm{sec}$ and (b) 100e/Å2.sec. The (c) shows higher electron dose rate gives shorter delay time for hydrogen bubble formation (d) shows that a nano probe of high dose rate electron beam can stimulate phase transformation from insulator phase to metal phase in a VO2 nanowire at room temperature.
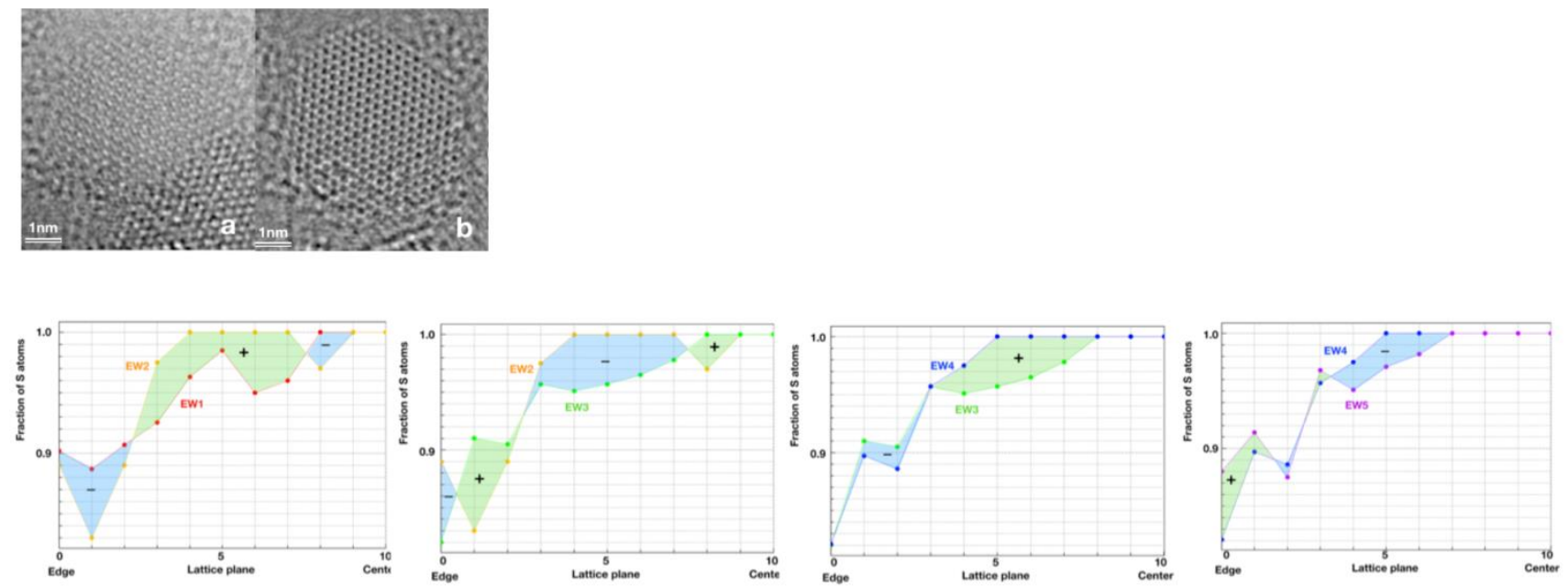

(c)

Figure 2. (a) modulus (b) phase of an exit wave function of MoS2 nanoparticle (c) The evolution of the defect $\mathrm{S}$ vacancy as a function of time. The time resolution is about 20 second.

\section{References}

[1] S. Helveg, C.F. Kisielowski, J.R. Jinschek, P. Specht, G. Yuan, H. Frei, Micron 68 (2014) 176-185.

[2] C. Kisielowski, P. Specht, S.M. Gygax, B. Barton, H.A. Calderon, J.H. Kang, R. Cieslinski, Micron 68 (2014) 186-19

[3] Y. Lu, .etc, F.-R. Chen and M. L. Sui, ACS Nano 2017, 11, 8018-8025 , DOI: 10.1021/acsnano.7b02656

[4] Y. Lu, .etc, F.-R. Chen and M. L. Sui, NATURE COMMUNICATIONS | (2018)9:2752 | DOI: 10.1038/s41467-018-05144-1 
[5]Z. Zhang, etc., F.-R. Chen and M. L. Sui, Nano Lett. 2017, 17, 851-855, DOI: 10.1021/acs.nanolett.6b04118

[6]Dirk Van Dyck, Joerg R. Jinschek \& Fu-Rong Chen, NATURE, 486 (2012) 243

[7] F.-R. Chen, D. Van Dyck \& C. Kisielowski, NATURE COMMUNICATIONS | 7:10603 | DOI: 10.1038/ncomms 10603 